\title{
A new type of solution of the Schrödinger equation on a self-similar fractal potential
}

\author{
N L Chuprikov and O V Spiridonova \\ Tomsk State Pedagogical University, 634041, Tomsk, Russia
}

\begin{abstract}
.
Scattering a quantum particle by a self-similar fractal potential on a Cantor set is investigated. We present a new type of solution of the functional equation for the transfer matrix of this potential, which was derived earlier from the Schrödinger equation.

PACS numbers: 03.65.Ca, 03.65.Xp
\end{abstract}


In this Letter we address the model [1, 2] of scattering a quantum particle by a self-similar fractal potential (SSFP) given on a Cantor set. This scattering problem is, perhaps, the most simple one to allow studying the influence of the scale invariance of ideal deterministic fractals on physical processes in continuous media to involve such fractal structures.

Note that the sharp attenuations, found in [1], in the spectrum of probability waves transmitted through this ideal fractal potential have also been observed experimentally (see [3]) in the transmission spectrum of electromagnetic waves propagating through a real fractal medium (a numerical modelling for the corresponding pre-fractals see in [4]). However, the problem is that the model [1, 2] remans incomplete in some respects. In this Letter we present a new type of solution to the Schrödinger equation on the SSFP, in addition to two types presented in [1, 2].

So, let $V(x)$ be a SSFP on the generalized Cantor set in the interval $[0, L]$; each level of the SSFP consists of $N(N \geq 2)$ SSFPs of the next level whose width is $\alpha$ times smaller than that of the former (see [2]). Let also $W$ be a power of the SSFP, that is, its total area: $W=\int_{-\infty}^{\infty} V(x) d x$. In line with [1, 2], for a particle with a given energy $E\left(E=\hbar^{2} k^{2} / 2 m\right)$, the transfer matrix $Z(\phi)(\phi=k L)$ of the SSFP must obey the functional equation

$$
\begin{aligned}
& \mathbf{Z}(\phi)=\mathbf{Z}(\alpha \phi)[\mathbf{D}(\gamma \phi) \mathbf{Z}(\alpha \phi)]^{N-1} ; \\
& \mathbf{Z}(\phi)=\left(\begin{array}{cc}
q(\phi) & p(\phi) \\
p^{*}(\phi) & q^{*}(\phi)
\end{array}\right), \quad \mathbf{D}(\phi)=\left(\begin{array}{cc}
e^{i \phi} & 0 \\
0 & e^{-i \phi}
\end{array}\right) ; \\
& q(\phi)=\frac{1}{\sqrt{T(\phi)}} \exp [-i J(\phi)], \quad p(\phi)=\sqrt{\frac{R(\phi)}{T(\phi)}} \exp \left[i\left(\frac{\pi}{2}+F(\phi)\right)\right]
\end{aligned}
$$

$\gamma=\frac{\alpha-N}{\alpha(N-1)} ; R=1-T ; T(\phi), J(\phi)$ and $F(\phi)$ are, respectively, the transmission coefficient and phase characteristics of the SSFP; here $F=0$ for the SSFP-barriers and $F=\pi$ for the SSFP-wells (see [2]).

As it has turned out, Eq. (11) not uniquely determines the transfer matrix of the SSFP. Two different types of solutions of this equation have been presented in [1, 2]. Remind that the first type was obtained for any values of $W, \alpha$ and $N$ to characterize the SSFP. In this case, for small values of $\phi, \sqrt{T(\phi)} \sim y(\phi) \sim \phi^{s}$, where $s$ is the fractal dimension of the Cantor set: $s=\ln (N) / \ln (\alpha) ; y=\frac{\pi}{2}-J$. The second type of solutions exists only for the SSFP-barriers, if $W=\frac{3 N \hbar^{2}}{m L}$. For this type, $\sqrt{T(\phi)} \sim y(\phi) \sim \phi$ for small values of $\phi$.

In this Letter we present a new (third) type of solutions (found by Chuprikov), with a cardinally different behavior of the tunneling parameters in the asymptotic region. Namely, in this case, for small values of $\phi$ we have

$$
\begin{aligned}
& T(\phi)=\left\{1+\cosh ^{2}[\omega(\ln (\phi))] \sinh ^{2}\left(c \phi^{-s}\right)\right\}^{-1}, \\
& J(\phi)=\arctan \left\{\sinh [\omega(\ln (\phi))] \tanh \left(c \phi^{-s}\right)\right\},
\end{aligned}
$$


where $c$ is a nonzero constant; $\omega$ is a nonzero real-valued function to obey the condition, $\omega[\ln (\phi)]=\omega[\ln (\phi)+\ln (\alpha)]$.

To extend this solution onto the whole $\ln (\phi)$-axis, one has to use the recurrence relations (18) and (19) presented in [2]. As in [1, 2], we display here $\ln (R / T)$ versus $\ln (\phi)$. Figures (1)-(6) show this function for three values of $\omega-1,10$ and 15 - and three values of $c-0.001,0.01$ and 0.1 . As is seen, there are three regions on the $\ln (\phi)$-axis, with a qualitatively different dependence of $\ln (R / T)$ :

in the left region

$\ln \ln (R / T) \sim \ln (2|c|)-s \ln (\phi) ;$

in the right one

$\ln (\widetilde{R / T}) \sim-2 s \ln (\phi)$,

where $\widetilde{R / T}$ is the envelop of $R(\phi) / T(\phi)$.

As regards the middle region, two qualitatively different types of changing this function are possible here. For $\omega=1$ and all three values of $c$ (see figures (1) and (2)) $\ln (R / T) \sim-2 s \ln (\phi)$.

Such behavior also occurs for $\omega=10$ and $c=0.001$ (see figures (3) and (4)). At the same time, for $\omega=15$ and all three values of $c$ (see figures (5) and (6)), as well as for $\omega=10$ and $c=0.1$ (see figures (3) and (4)) we have

$\ln (R / T) \sim-2 \ln (\phi)$.

Note that the right region appears for all three types of solutions (see [2]). As regards the left one to follow from (2), such a behavior is a distinctive feature of the third type of solutions.

It is also important to note here that for the solutions of the first and third types the phase path of the wave inside the out-of-barrier regions (i.e., in the regions where the potentials are equal to zero) is infinitesimally small in comparison with the wave path in the barrier regions. This feature distinguishes these types of solutions from the second one.

A simple analysis shows that the tunneling parameters are non-differentiable functions at the point $\phi=0$, when $\omega$ depends on $\phi$. Figure 7 shows the function $R(\phi) / T(\phi)$ for this case.

So, there are at least three types of the transfer matrices of the SSFP. As is seen, though all of them are nonzero only on the Cantor set, i.e., the set of zero measure, we deal with different potentials. The Cantor set is a non-countable one, and, thus, it yet provides a much enough room for setting potentials with such different scattering properties.

Of course, in this case, it is of great importance is to find the sequences of prefractals to lead to the SSFPs, when the generation number of pre-fractals tends to infinity. Additionally, another open question regarding the model is that the parameters to enter the third type of solutions remain to be connected to the SSFP parameters. 
A new type of solution of the Schrödinger equation on a self-similar fractal potential 4

\section{References}

[1] Chuprikov N L 2000 J. Phys. A: Math. Gen. 334293

[2] Chuprikov N L and D N Zhabin 2000 J. Phys. A: Math. Gen. 334309

[3] Takeda M W, Kirihara S, Miyamoto Y, Sakoda K and Honda K 2004 Phys. Rev. Lett. 92093902

[4] Honda K and Otobe Y 2006 J. Phys. A: Math. Gen. 39 L315

\section{Figure captions}

$\backslash$ Figure $\{\backslash$ label\{fig1\}The $\$ \backslash \ln (\backslash p h i) \$$-dependence of $\$ \backslash \ln (\mathrm{R} / \mathrm{T}) \$$ for $\$ s=0.5 \$, \$ c=0.001 \$$ and $\$ \backslash$ omega=1\$; bold full curve - $\$ N=2 \$$, thin full curve - $\$ N=4 \$$; points show the asymptote $\$ 13-2 s \backslash \ln (\backslash p h i) \$$.

$\backslash$ Figure $\{\backslash$ label\{fig2\}The $\$ \backslash \ln (\backslash \mathrm{phi}) \$$-dependence of $\$ \backslash \ln (\mathrm{R} / \mathrm{T}) \$$ for $\$ N=3 \$$, $\$ \backslash$ alpha=13\$ and $\$ \backslash$ omega=1\$; broken curve - $\$ c=0.1 \$$; thin full curve - $\$ c=0.01 \$$; bold full curve $\$ c=0.001 \$$; circles show the asymptote $\$ 10-2 s \backslash \ln (\backslash p h i) \$$.

$\backslash$ Figure $\{\backslash$ label $\{$ fig3\}The $\$ \backslash \ln (\backslash$ phi $) \$$-dependence of $\$ \backslash \ln (\mathrm{R} / \mathrm{T}) \$$ for $\$ \mathrm{~s}=0.5 \$, \$ \mathrm{c}=0.001 \$$ and $\$ \backslash$ omega=10 $\$$; bold full curve - $\$ N=2 \$$, thin full curve - $\$ N=4 \$$; points show the asymptote $\$ 3-2 s \backslash \ln (\backslash p h i) \$$.

$\backslash$ Figure $\{\backslash$ label\{fig4\}The $\$ \backslash \ln (\backslash \mathrm{phi}) \$$-dependence of $\$ \backslash \ln (\mathrm{R} / \mathrm{T}) \$$ for $\$ \mathrm{~N}=3 \$$, $\$ \backslash$ alpha=13\$ and $\$ \backslash$ omega=10\$; broken curve - $\$ c=0.1 \$$; thin full curve - $\$ c=0.01 \$$; bold full curve $\$ c=0.001 \$$; points show the asymptote $\$ 8-2 \backslash \ln (\backslash p h i) \$$; circles show the asymptote $\$ 4-2 s \backslash \ln (\backslash p h i) \$$.

$\backslash$ Figure $\{\backslash$ label\{fig5\}The $\$ \backslash \ln (\backslash p h i) \$$-dependence of $\$ \backslash \ln (\mathrm{R} / \mathrm{T}) \$$ for $\$ \mathrm{~S}=0.5 \$, \$ \mathrm{c}=0.001 \$$ and $\$ \backslash$ omega=15; bold full curve - $\$ N=2 \$$, thin full curve - $\$ N=4 \$$; points show the asymptote $\$ 5-2 \backslash \ln (\backslash \mathrm{ph} i) \$$.

$\backslash$ Figure $\{\backslash$ label\{fig6\}The $\$ \backslash \ln (\backslash \mathrm{phi}) \$$-dependence of $\$ \backslash \ln (\mathrm{R} / \mathrm{T}) \$$ for $\$ N=3 \$$, $\$ \backslash$ alpha=13 and $\$ \backslash$ omega=15\$; broken curve - $\$ c=0.1 \$$; thin full curve - $\$ c=0.01 \$$; bold full curve $\$ c=0.001 \$$; points show the asymptote $\$ 8-2 \backslash \ln (\backslash p h i) \$$; circles show the asymptote $\$ 4-2 s \backslash \ln (\backslash p h i) \$$.

$\backslash$ Figure $\{\backslash$ label\{fig7\}The $\$ \backslash \ln (\backslash p h i) \$$-dependence of $\$ \backslash \ln (\mathrm{R} / \mathrm{T}) \$$ for $\$ s=0.5 \$, \$ c=0.001 \$$ and $\$ \backslash$ omega=15 $\backslash$ left $[\backslash \sin \backslash \operatorname{left}(2 \backslash$ pi \frac$\{\backslash \ln (\backslash p h i)\}\{\backslash \ln (\backslash$ alpha $)\} \backslash r i g h t)+1.001 \backslash r i g h t] \$$; bold full curve - $\$ N=2 \$$, thin full curve - $\$ N=4 \$$; points show the asymptotes $\$ 5-2 \backslash \ln (\backslash p h i) \$$ and $\$ 14-2 s \backslash \ln (\backslash p h i) \$$. 


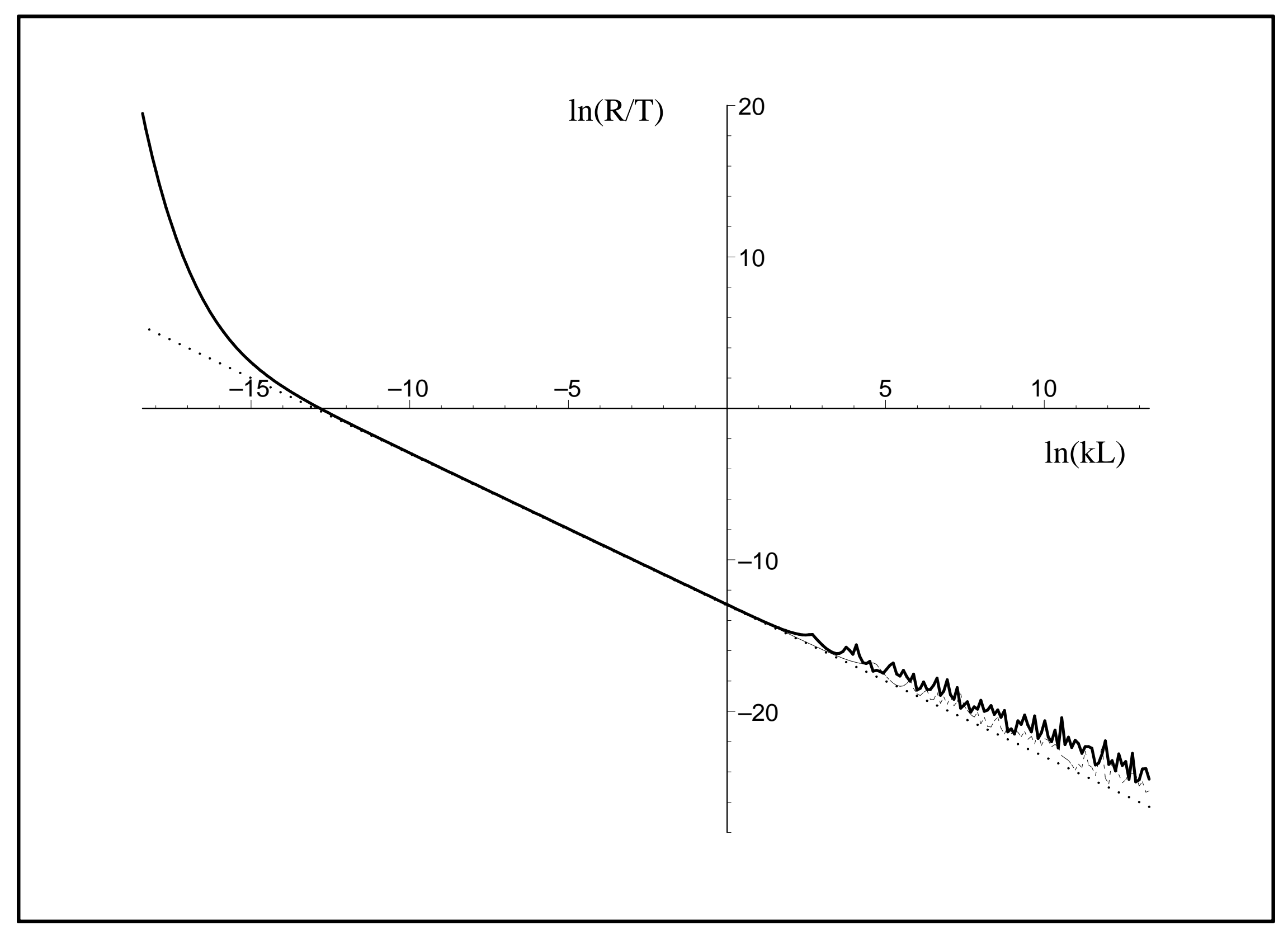




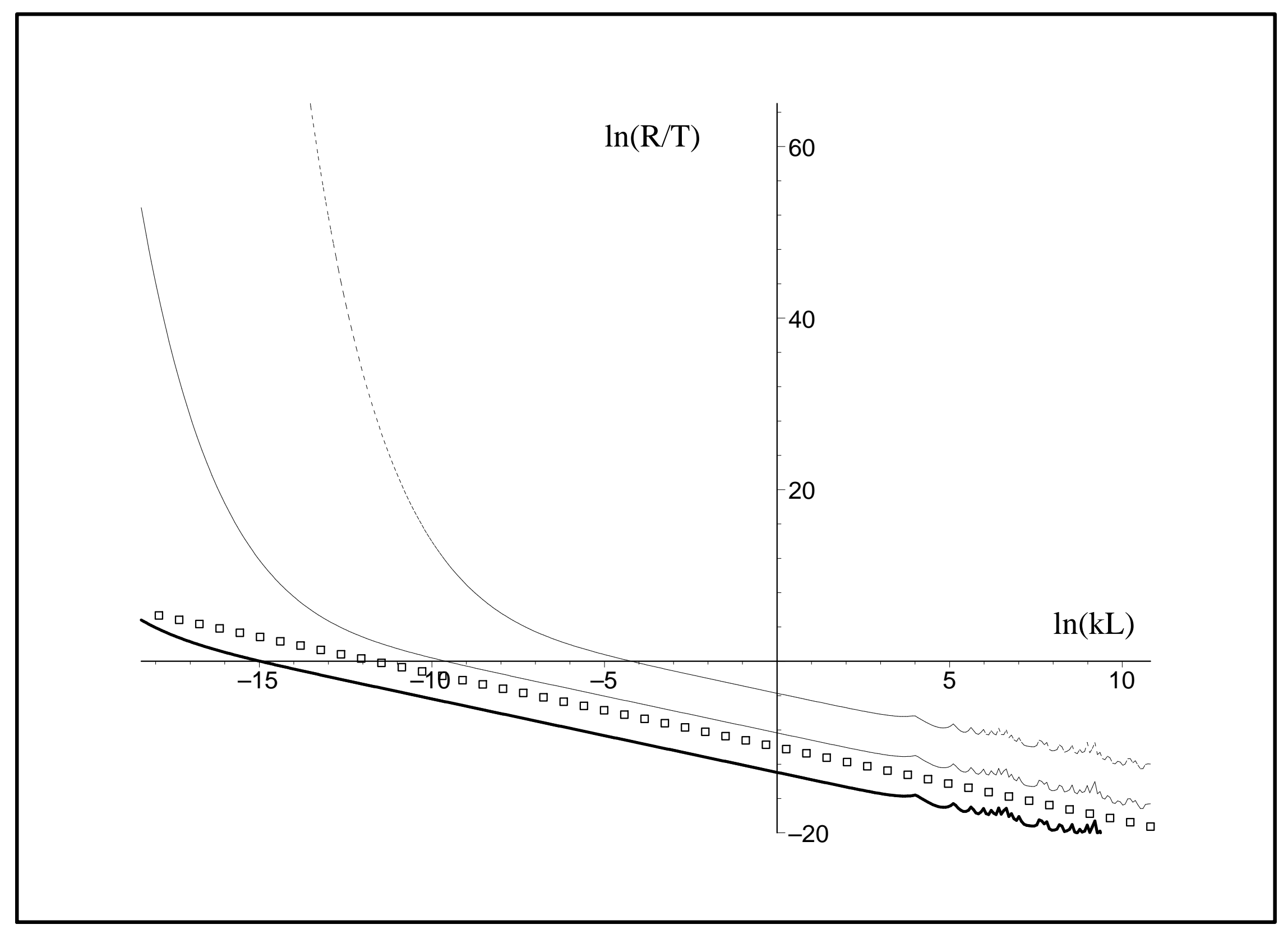




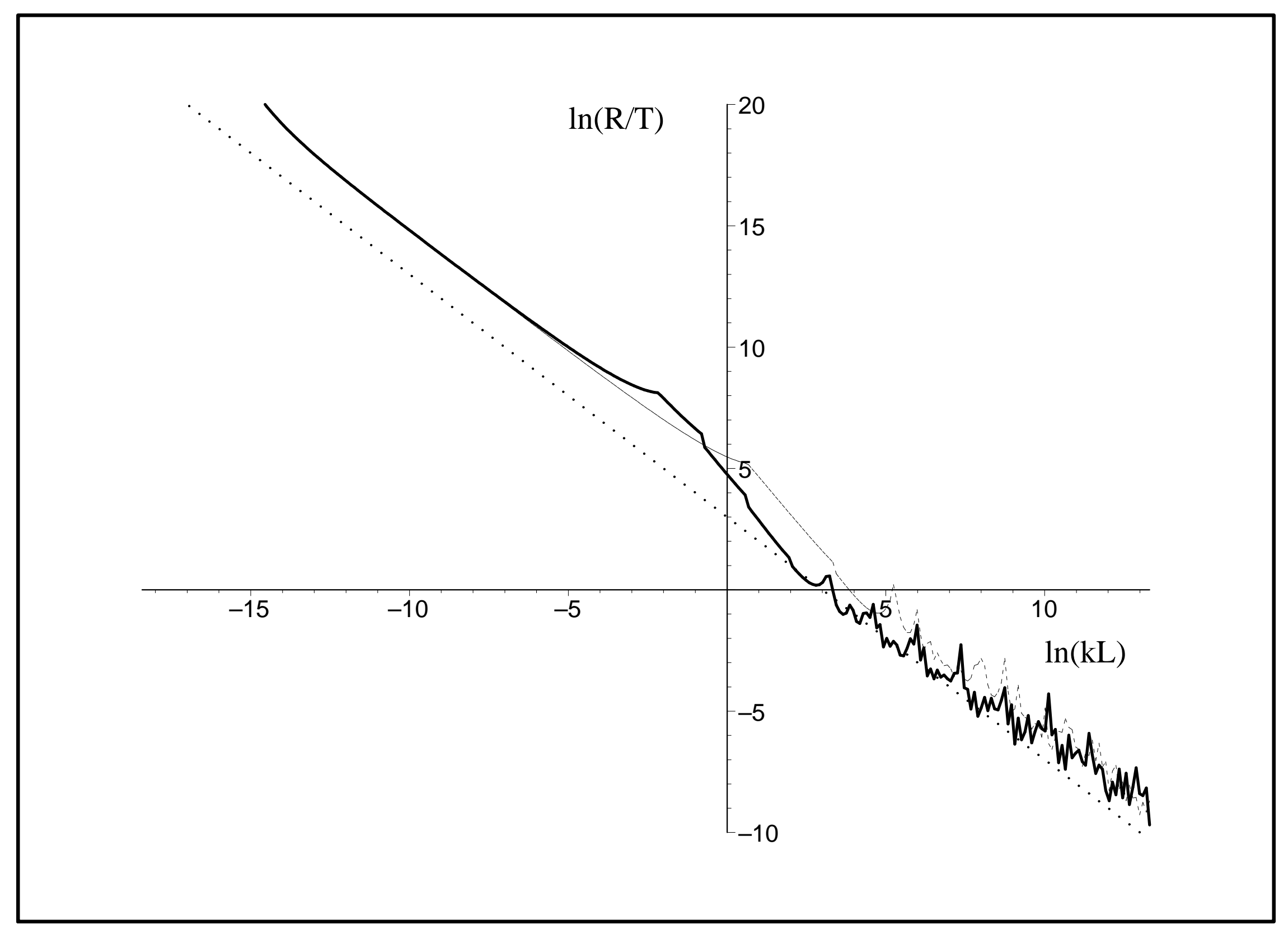




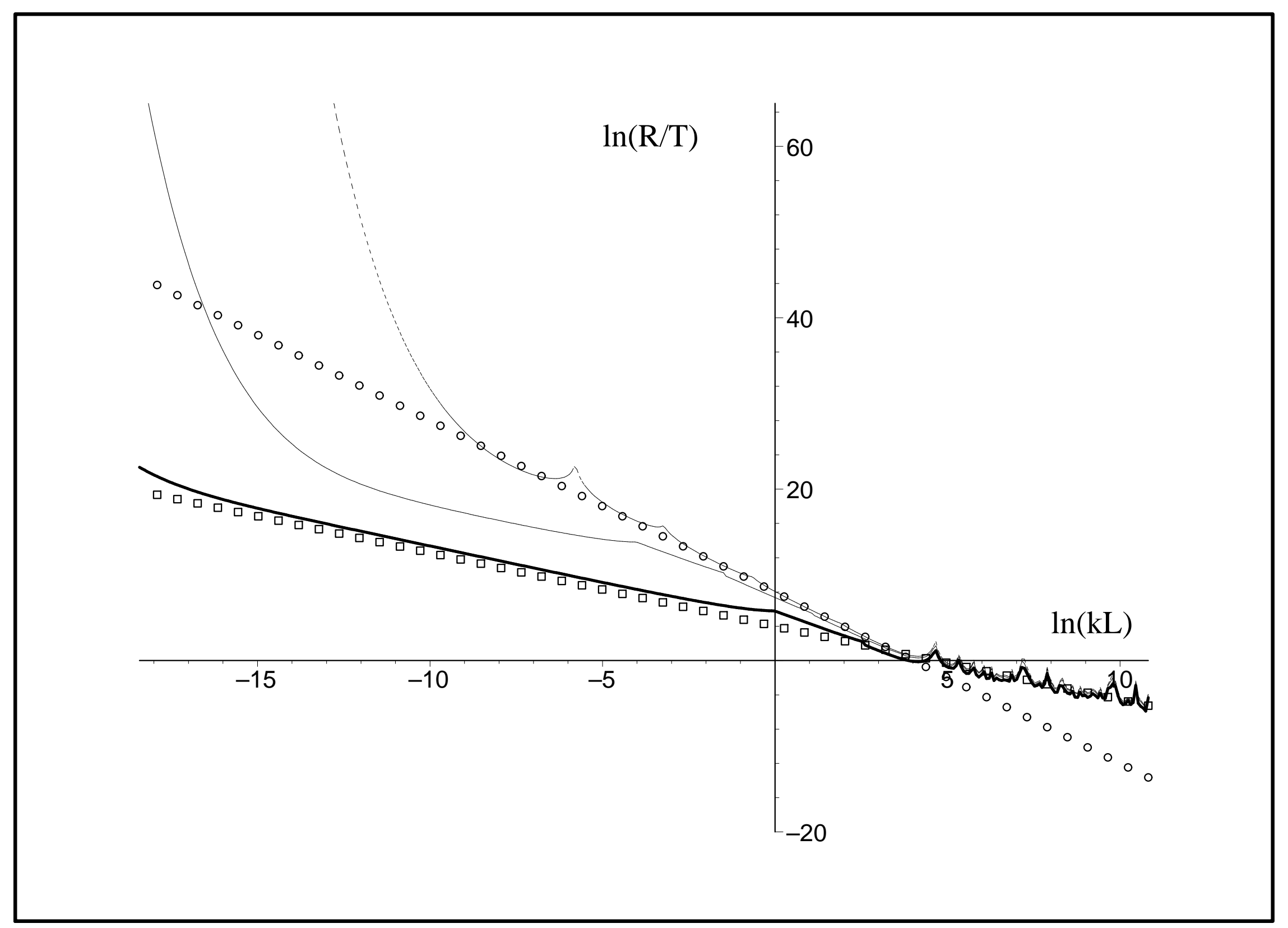




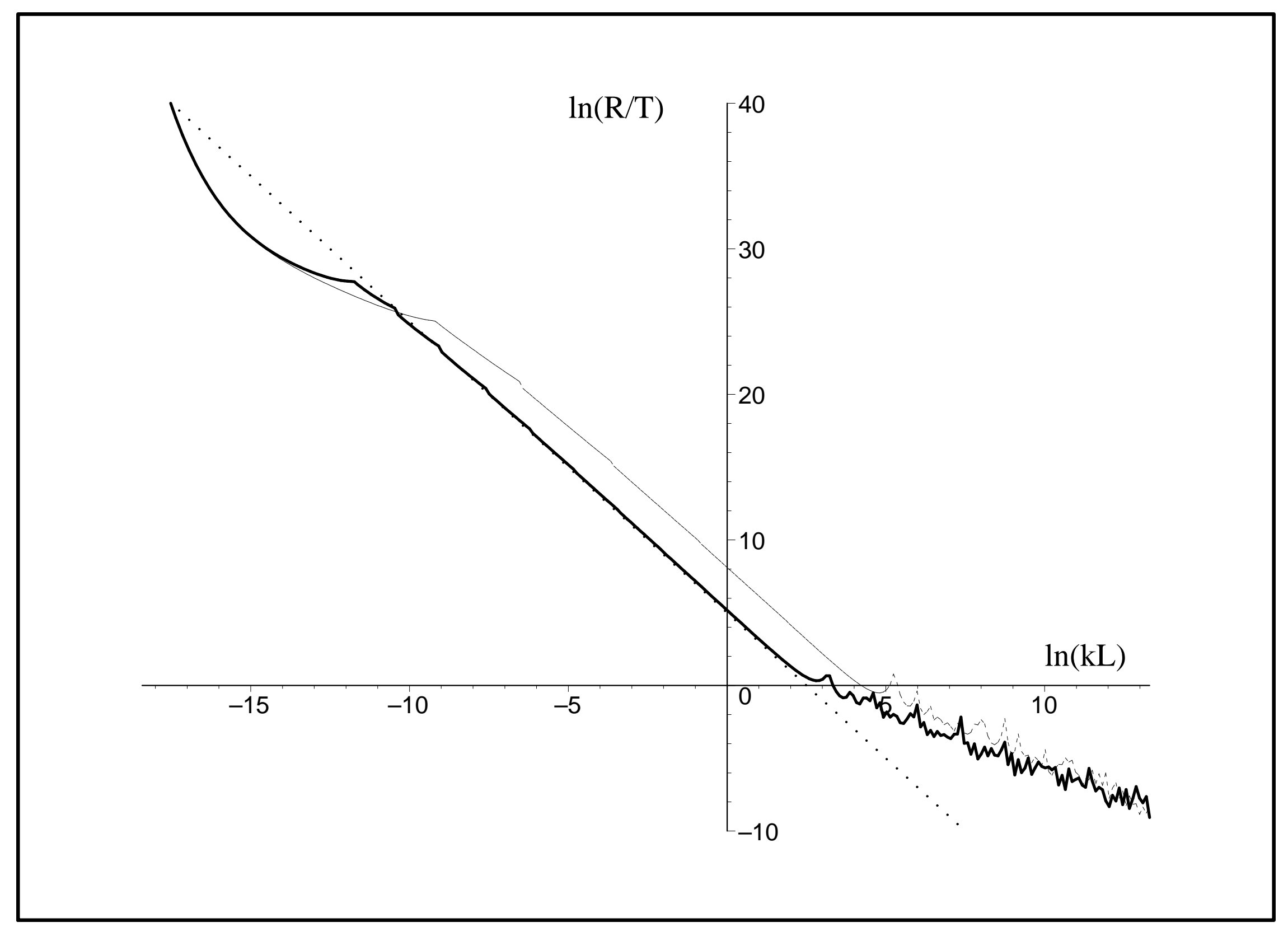




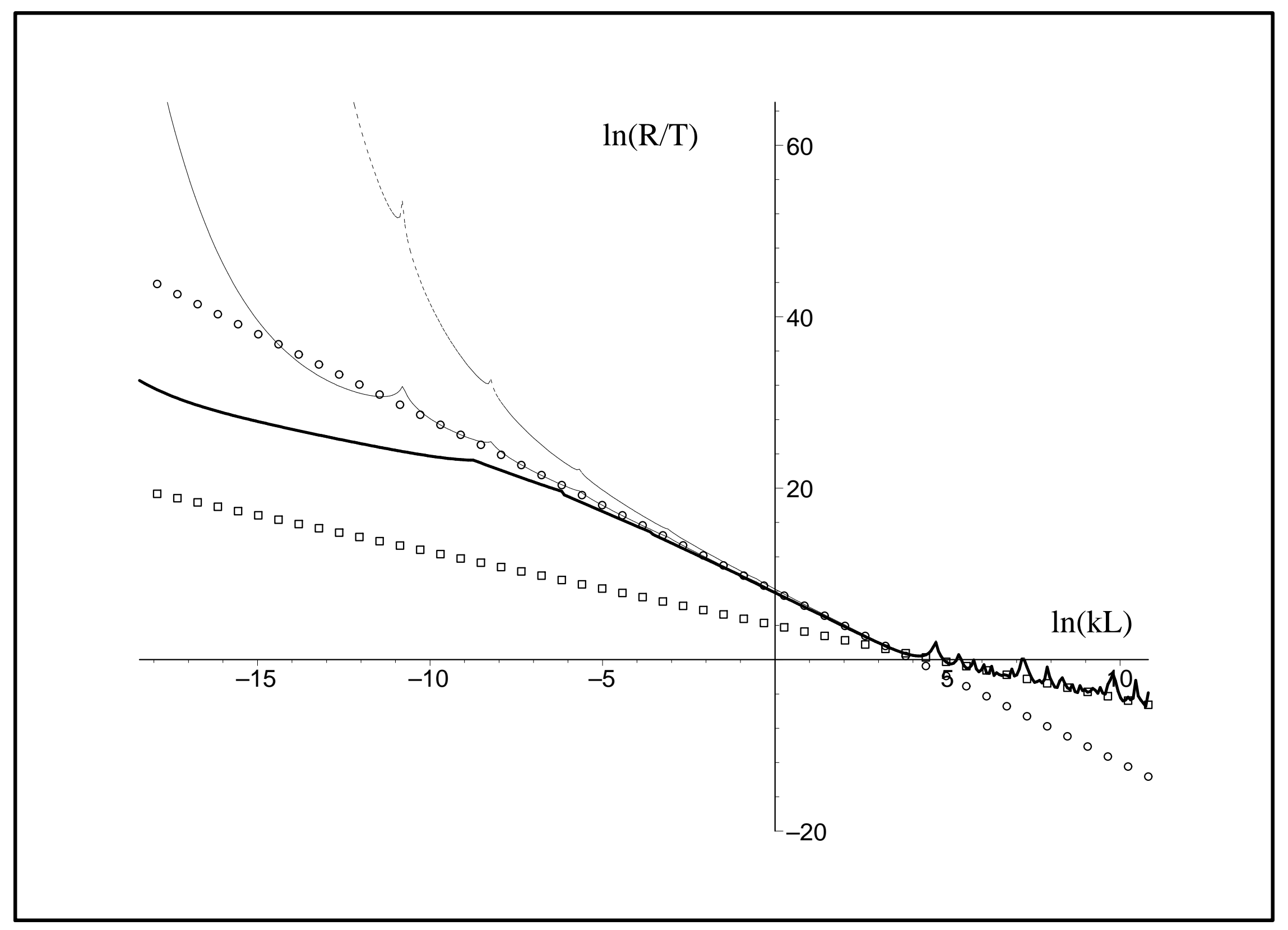




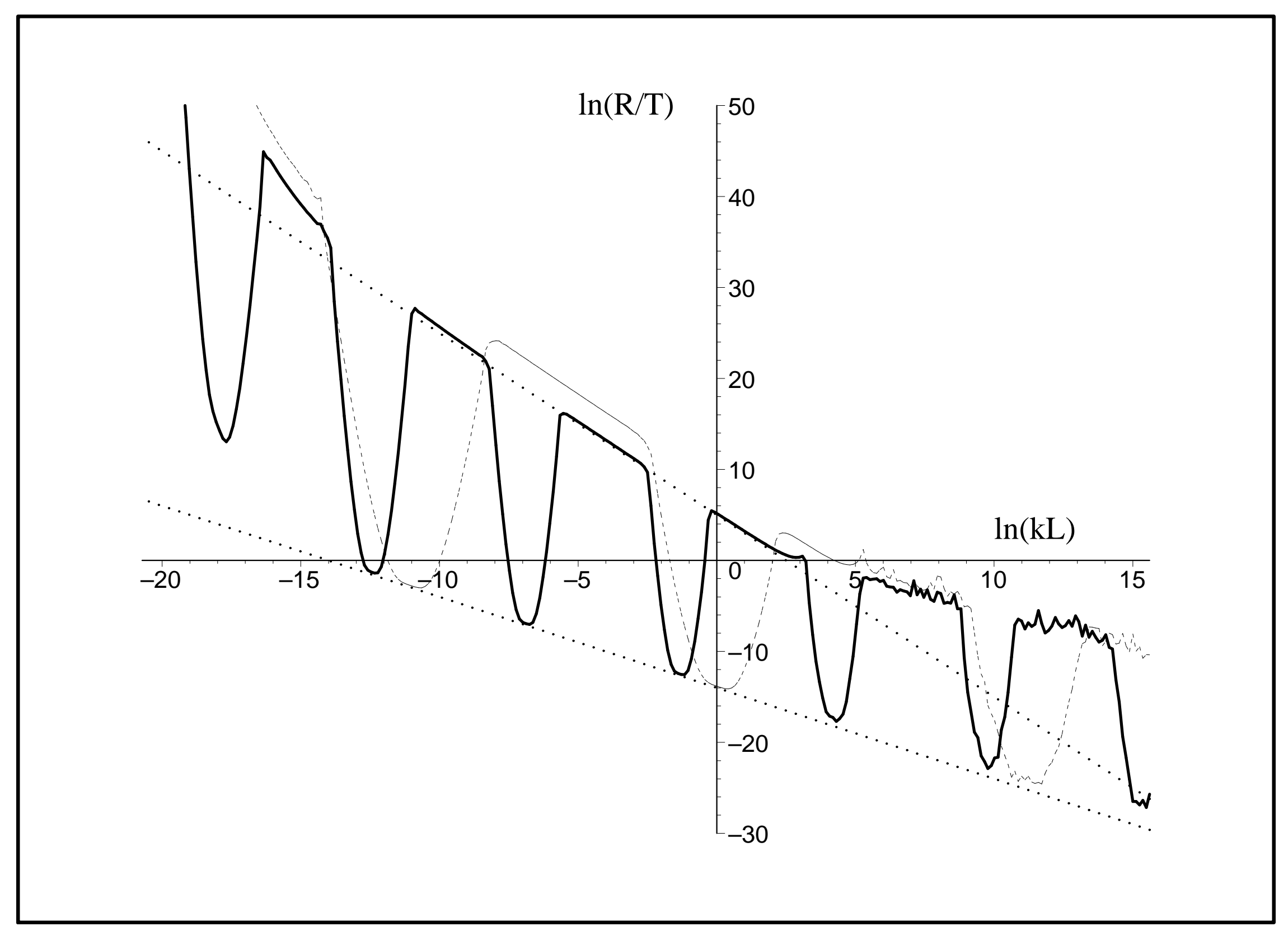

